\title{
RESEACH ON ASP.NET AJAX FRAMEWORK AND ITS APPLICATION IN WEB DEVELOPMENT
}

\author{
Qian Wang ${ }^{a,{ }^{*}}$, Lili $\mathrm{Yu}^{\mathrm{b}}$ and Jinzhen Ping ${ }^{\mathrm{c}}$ \\ Department of Software Engineering, Shijiazhuang Information Engineering Vocational College, \\ Shijiazhuang, 050035, China \\ a25734476@qq.com, b76192918@qq.com, ${ }^{\mathrm{c}} 41472796 @ q q . c o m$, *corresponding author
}

\author{
Keywords: AJAX; ASP.NET; Web Program
}

\begin{abstract}
Web page requests is the common way for applications to implement human-computer interaction, but full page refreshing of the page reduces the effects of users 'experience, increases the pressure of servers and slows down the speed of program response. Partial update pages with AJAX asynchronous transfer technology can increase the efficiency of program interactions and give user a friendly program interface. Microsoft's ASP. NET AJAX framework can greatly reduce the difficulty of programmers in developing AJAX applications. This paper introduces AJAX engine principle and ASP. NET AJAX framework firstly, and then describes ASP. NET AJAX configuration in the Web site, at the end we imported AJAX application in the design of online examination system in order to achieve asynchronous partial update, therefore a friendly user interactive interface is brought about.
\end{abstract}

\section{Introduction}

AJAX called "Asynchronous JavaScript and XML" was first proposed by Jesse James Garrett, the core of which is the use of AJAX asynchronous interaction patterns, reducing server load and improving application performance [1]. It is a mature set of seamless collection of old technology, including: using CSS and XTML standardized presentations; Dynamic display and interaction using DOM; Data exchange and processing using XML and XSLT; using XML Http Request for asynchronous data reading; Finally, JavaScript bindings and processing data. Introducing AJAX into traditional web applications technology advantages as embodied in the following several aspects: (1) AJAX separates data and presentation which contributes to a division of labor, and reduces web application error caused by non-technical staff to modify the page; (2) AJAX access data according to the demands, reduces redundancy request furthest, reducing the total amount of downloaded data, thus reducing the load on the server; (3) AJAX is a rich client technology, some of the previous work is transferred to the client from the server, using the spare capacity of the client to handle, reduce the burden of data processing at the server; (4)Reduce consumption of bandwidth; (5) Updating page without refreshing, reducing the psychological and actual waiting time, avoid the white screen.

The advantages of AJAX are very significant, but to build AJAX-style Web applications manually is difficult, and Microsoft's ASP.NET AJAX perfectly encapsulates AJAX technology, is a visual AJAX application framework. It did a lot of object-oriented expansions for JavaScript, coupled with the integrated ASP. NET development platform, and reduces the difficult level for AJAX programmers to develop large programs. Therefore, The Online examination system based on ASP.NET platform introduces ASP. NET AJAX framework, you can easily develop AJAX functionality, improved the issues of traditional Web model such as the slow reaction of clients, refreshing frequently and so on.

\section{Research on the ASP.NET AJAX Framework}

Principle of AJAX Engine. AJAX is equivalent to adding a middle layer between the user and the server that allows users' operation and the server response to asynchronously. Not all of user requests are submitted to the server to be processed. Some operations don't need to reload the entire page such as editing the data in the memory, page navigation and data check. This kind of process can be 
delivered to the AJAX engine. When the page need to read the new data from the server ,the AJAX engine then send an asynchronous request to the server [2]. So the user does not need to wait or request response, you can perform other operations. The comparison of the two methods is shown in Figure 1.

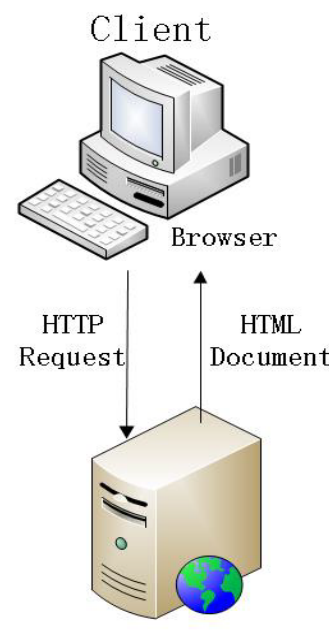

Traditional Web Work

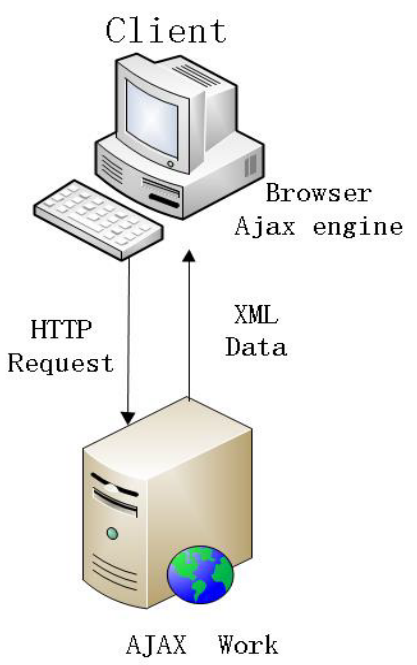

Figure 1 Comparison between the traditional work and AJAX work

According to the Figure1, the traditional Web work is very simple. A HTTP request is sent by the browser, and then processed after the server receives the request, and then the server returns a HTML/XTML page to the client browser and displays it to the user. This way of working is bound to the user waits, while AJAX has added a engine between the client's browser and the Web server. The engine is downloaded to the client browser when the browser communicates with the server for the first time until the completion of client-side and server-side communication.

ASP. NET AJAX Architecture. ASP. NET AJAX consists of two parts: the client-script libraries and server components [3]. This two-part integrated to provide a robust development framework. To implement ASP. NET AJAX can take advantage of server; can also take advantage of client implementation, or a combination. In essence, the server implementations described here need client script; any AJAX-based page needs some client JavaScript code to handle the browser's document object model (DOM) and any application-specific extensions. However, ASP. NET programmer doesn't have to writing script code, framework specifically designed for scripting code that can be generated as the output of a server control.

ASP. NET AJAX Server Architecture. ASP. NET AJAX server controls consist of server and client code, and integrated codes can produce rich client behavior. When the AJAX controls are added to the ASP.NET page, the page will send the supported client script to the browser for AJAX functionality. The following are commonly used ASP.NET AJAX server control: ScriptManager manages all components on the page as well as partial page updates, and generates client proxy script to be able to access Web services in JavaScript. UpdatePanel control dependent on the ScriptManager control and PageRequestManager client class, including local content. UpdateProgess control used to display the progress information of the UpdatePanel control is refreshed. Timer control is sent to the server at fixed time intervals either synchronous or asynchronous requests.

ASP. NET AJAX Client Architecture. ASP. NET AJAX client-script library consist of JavaScript (.js) files, and provide object-oriented JavaScript features [4], consists of the following sections: the implementations of JavaScript and DOM in the browser compatible have some minor differences in each browser, one of the most obvious is the XMLHttpRequest object. Remove the differences between the various browsers available to other unified application programming interface. Some framework basic class library has been implemented. It also has network access layer, a user interface basic class libraries, and ASP. NET AJAX client components.

ASP. NET AJAX Web Site Configuration. After installed ASP. NET AJAX core components successfully; you can add AJAX functionality to an existing system. To an existing site, usually there is some value to be retained; normally you need to add a new ASP. NET AJAX configuration 
elements to an existing configuration file manually. The configuration file includes $<$ configSections $>$ elements, <controls > elements, <assemblies > elements, <httpHandlers $>$ elements, <httpModules $>$ elements, <System. web. Extensions $>$ element and $<$ system. Webserver $>$ elements. When the configuration is complete, we can add the AJAXExtensionToolbox.dll into the VS toolbox manually. Then the ASP.NET AJAX server controls can be seen on the left side bar of the project, as shown in Figure 2.

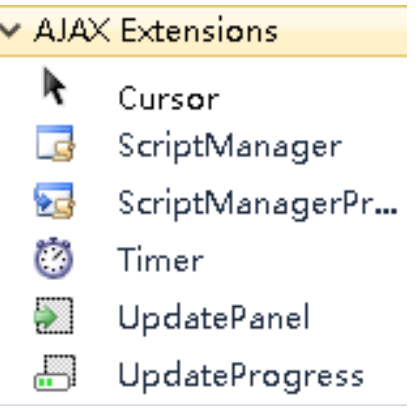

Figure 2 ASP.NET AJAX server control

ASP. NET AJAX Application in the Online Examination System. Following a case of online examination system design. In online examination system, the answer page is placed in a frames page. Because the students need to switch between the various questions, the traditional page refreshing will refresh the test request set and the page object will refress. But Only the test records need to be refreshed. Now AJAX with asynchronous transfer can record update, thereby improving processing speed, reduce the burden of server.

To solve the above problems, we add a ScriptManager and UpdatePanel controls, they can achieve asynchronous partial update of the page.

Add the ScriptManager Control. In each of the page supported ASP. NET AJAX has the exactly only one ScriptManager control, other implementations of AJAX features depend on this control. Due to user requests, the master page and content page generates dynamically a page class, so we add a ScriptManager control on the master page, and there is no need to add a ScriptManager control to the page. We just need to add the UpdatePanel control.[5] When the EnablePartialRendering property of the ScriptManager control is true, you can implement asynchronous partial update for the page, and the default value is true. In addition, when you add a ScriptManager control on the master page, ensure that add it in the form elements, except for contentplaceholder control.

Add the UpdatePanel Control. UpdatePanel controls are added in the content page. Click the ASP. NET server control within the UpdatePanel and sent an asynchronous post back request, the server returns partial refresh content after a request is received.

- UpdateMode property

UpdatePanel update mode whose default value is Always. But this property is set to conditional. UpdatePanel updates only when actions trigger the echo in its internal controls (when the ChildrenAsTriggers property is true), or start a defined trigger updates.

- Triggers property

In ASP. NET AJAX, there are two types of Triggers: AsyncPostBackTrigger and PostBackTrigger. AsyncPostBackTrigge is used to specify that a server-side control and its server-side event triggers as the asynchronous update of UpdatePanel trigger.[6] We need to set property control ID and server control events; PostBackTrigger is used to specify a server control in the UpdatePanel. It does not use asynchronous postback but still the traditional full-page postback.

Question ID for the CheckBoxList control is cbQuestionList and the trigger control event is SelectedIndexChanged, the following code:

$<$ triggers $>$

<asp: AsyncPostBackTrigger ControlID="cbQuestionList"

EventName="SelectedIndexChanged">

</asp: AsyncPostBackTrigger>

$<$ triggers $>$ 
- ChildrenAsTriggers property

When UpdatePanel property value is conditional, the internal control causes a postback can cause partial updates until the ChildrenAsTriggers property is set to True. After the Properties configuration is complete, the question page implements the asynchronous partial refresh and reduce the burden effectively on the server.[7]

\section{Summary}

ASP. NET AJAX framework and ASP. NET programming model integrates seamlessly. They built a bridge between application client script and Server languages. We add an ASP. NET AJAX server control into the online examination system and implement asynchronous local and refresh. Therefore make the communication convenient and bring enjoyable experience for users. It also reduce burden on the server caused by redundant requests and response.

\section{References}

[1] Kris Hadlock. Ajax---Web development, reusable components and patterns [M]. Beijing: Tsinghua University Press, 2007.

[2] Chen Feng min. Principle and development of Ajax [M]. Beijing: Tsinghua University Press, 2009.

[3] Wang Shihui, Chen Zhixiong, Zhu Rongzhao. ASP. NET AJAX applications in Web development [J]. Computer and information technology 2008 (Z1)

[4] Li Feng, Chao Yang. JavaScript development technology details [M]. Beijing: Tsinghua University Press, 2009.

[5] Microsoft's official Web site: http://www. asp. net/ajax.

[6] Yang Yanlan, Jin Xiaoxue, Ye Hua. ASP. NET AJAX framework and its application in Web development [J]. Computer applications and software, 2011 (6): 113-117.

[7] Huai Aiqin. AJAX technology in the research and application of Web system development [J]. Computer age 2010 (9): 78-80 